\title{
Solving Inaccuracies in the Heart Position and Orientation for Inverse Solution by Using Electric Information
}

\author{
Miguel Rodrigo $^{1}$, Andreu M. Climent ${ }^{1,2}$, Alejandro Liberos ${ }^{2}$, Ismael Hernández-Romero ${ }^{2,3}$, Angel \\ Arenal $^{2}$, Javier Bermejo ${ }^{2}$, Francisco Fernández-Avilés ${ }^{2}$, Felipe Atienza ${ }^{2}$ and Maria S. Guillem ${ }^{1}$ \\ ${ }^{1}$ ITACA Institute, Universitat Politècnica de València, Valencia, Spain \\ ${ }^{2}$ Hospital General Universitario Gregorio Marañón, Madrid, Spain \\ ${ }^{3}$ Rey Juan Carlos University, Fuenlabrada, Madrid, Spain
}

\begin{abstract}
Electrocardiographic Imaging (ECGI) has become an increasingly used technique for non-invasive diagnosis of cardiac arrhythmias, although the need for medical imaging technology to determine the anatomy hinders its introduction in the clinical practice. This work explores the ability of the L-curve curvature for identifying the location and orientation of the atrial surface inside the torso. Surface electrical signals from 31 mathematical models and four AF patients were used to estimate the optimal position of the atria inside the torso. The curvature of the L-curve from the Tikhonov method was measured after application of deviations in atrial position and orientation. Independent deviations in the atrial position were solved by finding the maximal L-curve curvature with an error of $1.7 \pm 2.4 \mathrm{~mm}$ in mathematical models and $9.1 \pm 11.5 \mathrm{~mm}$ in patients. Independent angular deviations were solved with an error of $5.8 \pm 7.1^{\circ}$ in mathematical models and $12.4^{\circ} \pm 13.2^{\circ}$ in patients. Under superimposed uncertainties in the 3 axis of translation and in the 3 axis of rotation, the error in location was of $2.3 \pm 3.2 \mathrm{~mm}$ and $6.4^{\circ} \pm 7.1^{\circ}$ in mathematical models, and 7.9 $\pm 10.7 \mathrm{~mm}$ and $10.0^{\circ} \pm 12.8^{\circ}$ in patients. The curvature of L-curve is a useful marker for emending the inaccuracies the cardiac location and would allow to combine torso and heart anatomies extracted from different image techniques.
\end{abstract}

\section{Introduction}

Electrocardiographic imaging (ECGI) is based on the resolution of the inverse problem of electrocardiography, making use of signals recorded by Body Surface Potential Mapping (BSPM) and the 3D anatomy of the torso and heart of the patients. All this information allows reversing the propagation model between the myocardial tissue and the patient's torso [1]. However, some inaccuracies in these data may appear when the CT/MRI images and the BSPM recordings are not obtained immediately one after another. This delay results in a mismatch in the heart location causing a loss in accuracy in the inverse-computed signals. Moreover, the heart position is subjected to other sources of movement artifacts, like respiration or ventricular contraction.

Tikhonov regularization is one of the methodologies used to solve the inverse problem and its optimization by the L-curve method allows the system to find a balance between the errors committed in the non-invasive signal estimation and the intrinsic numerical error of the inverse solution [1-2]. It has been observed that the worsening of the system conditions, such as the displacement of the heart within the torso, affects the L-curve shape. This paper evaluates whether the measurement of the L-curve deterioration quantified in terms of its curvature allows minimizing the uncertainties on the system conditions, namely the inaccuracies in the atrial position in a population of patients and models.

\section{Methods}

\subsection{Mathematical models}

A realistic 3D model of the atrial anatomy composed by 284,578 nodes and 1,353,783 tetrahedrons (673.4 \pm 130.3 $\mu \mathrm{m}$ between nodes) was used to simulate the atrial electrical activity during atrial fibrillation (AF) [3-4]. An ensemble of 31 different AF episodes was simulated with different degrees of complexity and different patterns of activation rates. For each simulation, a uniform mesh of unipolar EGMs was calculated at $1 \mathrm{~mm}$ from the epicardial surface by summing up all effective dipole contributions over the entire model. The ECG potentials on the torso model were calculated by solving the Forward Problem [5] in a mesh formed by 771 nodes, in which the atrial model was co-located within the torso by using the images from the Visual Human Project. White Gaussian noise was added to the synthetic ECG signals with a signal-to-noise ratio of $30 \mathrm{~dB}$. 


\subsection{Patient recording}

Multichannel electrocardiograms (ECGs) were recorded with 57 chest ECG leads by using the BSPM technique from $4 \mathrm{AF}$ patients admitted for ablation [6]. Atrial anatomy was obtained during the electrophysiological procedure by using the ablation catheter guided by an electro-anatomical navigation system that enabled atrial anatomy reconstruction. Nineteen AF signal segments from the 4 patients $(4.7 \pm 3.1$ segments/patient $)$ with duration of $5.6 \pm 2.1$ seconds analysed.

MRI images with a spatial resolution of $0.7 \times 0.7 \times 1.5 \mathrm{~mm}$ were acquired 2-3 days prior to the ablation procedure. Atria and torso anatomy were obtained by segmentation of MRI images. Additionally, the torso anatomy, together with the electrodes location was obtained by processing the conventional photographic images previously acquired. Anatomical models obtained with the different technologies were co-registered by using an algorithm based on rigid transformations guided by 8 points manually marked in both atrial models (4 PVs, LAA, RAA and SVC, IVC) or torso models.

\subsection{Inverse solution and L-curve}

We estimated the inverse-computed EGM from both patient recordings and mathematical models by using the zero-order Tikhonov's method [1]. The optimal regularization parameter was selected according to the Lcurve method, which choses the corner of the error norm vs. the solution norm for the different regularization parameters (see Fig. 1). This corner of the L-curve minimizes the overall error in the inverse solution and provides the ECGI solution that minimizes the extreme values on the solution that can be mostly attributed to the numerical errors involved in the transfer matrix inversion.

In order to explore the potential use of the L curve shape to estimate the location of the atria inside the thorax we varied the location and orientation of the atria both in our mathematical models and in our patient data and computed the maximum L-curve curvature under all tested model alterations. In Fig. 1, a sample case of the resolution of the ECGI with a modification in the model geometry is represented. The forward problem was solved with the atria at the "original location" and then the inverse problem was solved both for the atria at the original and "displaced" location ( $5 \mathrm{~cm}$ distance). With the departing EGMs (Fig. 1.A), a rotor can be identified in the posterior left atrial wall. In this case, inverse-computed phase maps calculated with the same geometries for the forward and inverse calculations do allow to identify the rotor at the same location, despite the differences that can be observed in potential maps, as shown in Fig. 1.C. However, ECGI maps obtained with a displacement in the atrial location (Panel D), no longer allow the identification of the rotor.

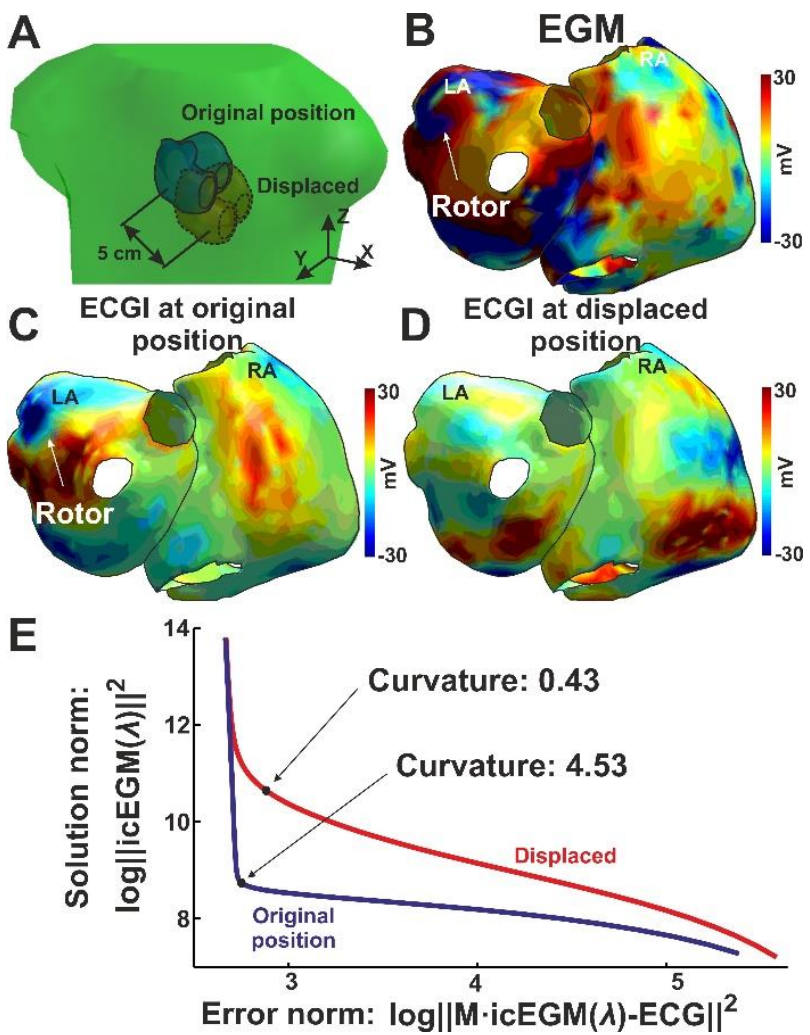

Figure 1. Illustration of the experimental set-up and inverse-computed examples. (A) Schematic view of the torso surface (green), atrial surface at the original position (blue) and atrial model at displaced position (red). Potential distribution for EGM (B), ECGI at the original position $(\mathrm{C})$ and ECGI at displaced $(5 \mathrm{~cm})$ position (D). (E) L-curves of the inverse solution for the original position (blue) and displaced positon (red).

\section{Results}

\subsection{Single axis displacement vs. curvature}

We first evaluated the curvature of the L-curve for displacements in the location of the atria inside the thorax in each of the $\mathrm{X}, \mathrm{Y}, \mathrm{Z}$ axis for the resolution of the inverse problem (Fig. 2). For displacements in the $\mathrm{X}$ axis of mathematical simulations (Fig. 2.A), the maximum curvature was found on average for the correct location of the atria. Indeed, 8 simulations showed their maximal curvature for $0 \mathrm{~mm}$ of displacement, and 2 simulations for $-5 \mathrm{~mm}$. Therefore, the actual location of the atrial anatomy could be estimated with a mean error of $1.0 \pm 2.1 \mathrm{~mm}$. Similar observations were made for displacements in the $Y$ and $\mathrm{Z}$ axes, with errors in the location of the atria of $3.0 \pm 2.6 \mathrm{~mm}$ and $1.0 \pm 2.1 \mathrm{~mm}$ for the displacement in the $Y$ and $\mathrm{Z}$ axes, respectively (Fig 2.C). The same experiments were conducted with patient data, in which the location of the atria, obtained my MRI, was altered in each of the X, $\mathrm{Y}$ and $\mathrm{Z}$ axes (Fig. 2). Again, the maximum curvatures 
were obtained at the actual location of the atria inside the thorax. If the location of the atria is defined as that with the largest L-curve corner curvature, then the observed errors were $5.8 \pm 5.1 \mathrm{~mm}, 7.2 \pm 8.9 \mathrm{~mm}$ and $14.2 \pm 16.1 \mathrm{~mm}$ for the $\mathrm{X}, \mathrm{Y}$ and $\mathrm{Z}$ axes, respectively $(9.1 \pm 11.5 \mathrm{~mm}$ on average).
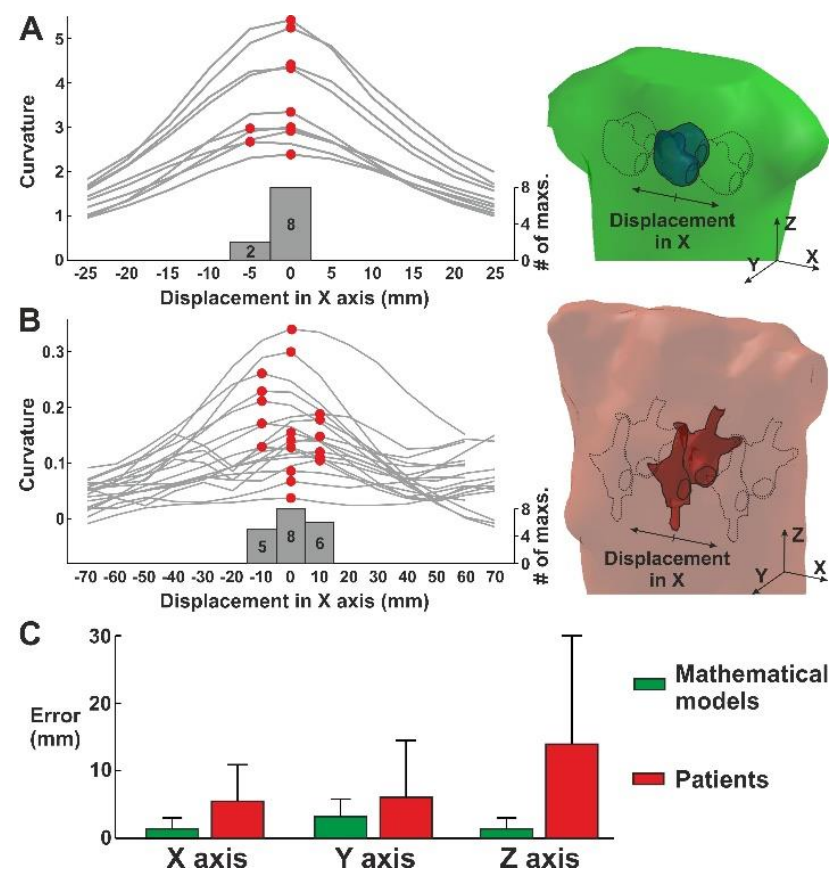

Figure 2. Estimation of atrial position under displacements in $X$ axis for (A) mathematical models and (B) patients. (C) Error in atrial position for independent displacements in the 3 axes.
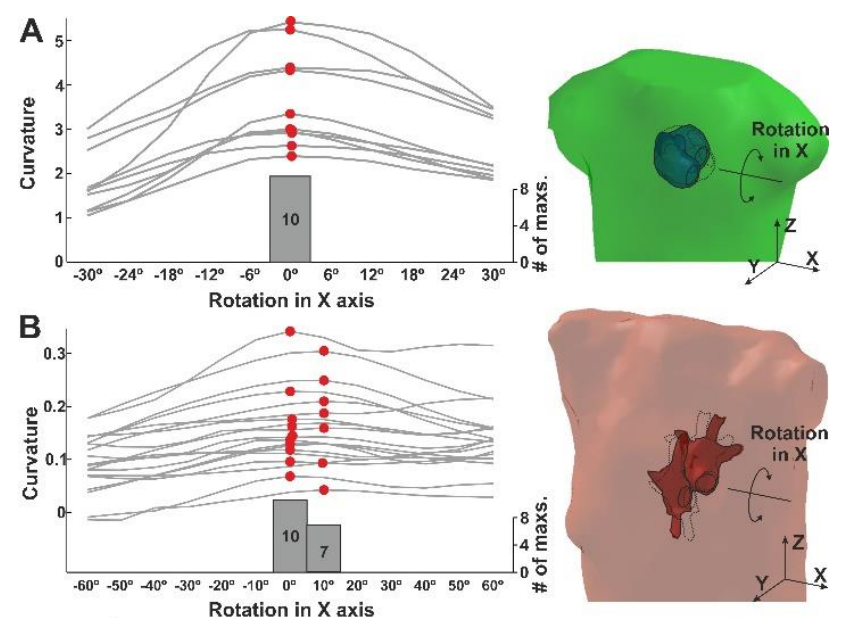

C

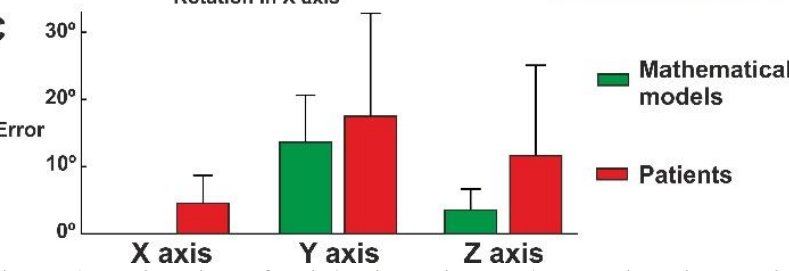

Figure 3. Estimation of atrial orientation under rotations in $\mathrm{X}$ axis for (A) mathematical models and (B) patients. (C) Error in atrial orientation for independent rotations in the 3 axes.

\subsection{Single axis rotation vs. curvature}

The second stage of the L-curve accuracy assessment was to evaluate its ability to properly detect a rotation in the atria inside the thorax. In Fig. 3, results of the inverse problem resolution after rotations between $-30^{\circ}$ and $30^{\circ}$ around each of the three axes are presented. In Fig. 3.A the curvatures obtained for each simulation affected by rotations in $\mathrm{X}$ axis are depicted, and all of them showed their maximal curvature of the L-curve in the actual location of the atria and, therefore, the error in rotation identification was $0.0 \pm 0.0^{\circ}$. Rotations in $\mathrm{Y}$ and $\mathrm{Z}$ axes, however, were solved with an error of $13.8 \pm 6.4^{\circ}$ and 3.6 $\pm 3.1^{\circ}$. In Fig. 3.B show the results from rotations of the atria inside the thorax of AF patients. Fig. 3.B shows the curvatures obtained after rotations around the $\mathrm{X}$ axis, which resulted in an error in the estimation of the angular position of the atria of $4.1 \pm 5.1^{\circ}$. Angular deviation in the $\mathrm{Y}$ and $\mathrm{Z}$ axes were solved in patients with an error in the angle detection of $18.3 \pm 15.0^{\circ}$ and $12.6 \pm 11.9^{\circ}$ respectively.

\subsection{Combined rotation and displacements}

Since errors in location and orientation of the atria inside the thorax can appear in combination, we then evaluated the potential use of the L-curve curvature for identifying these errors in a step-wise manner. For each of the mathematical models random rotations in the $\mathrm{X}, \mathrm{Y}$ and $\mathrm{Z}$ axes $\left(-30^{\circ}\right.$ to $\left.30^{\circ}\right)$ were applied and then we evaluated the curvature of the L-curve as a function of the distance from the actual location of the atria inside the thorax ( 0 to 45 mm, Fig. 4.A). It can be observed that the curvature showed its maximum values in the vicinities of the actual location, resulting in an error in the position estimation of $2.3 \pm 3.2 \mathrm{~mm}$. In patients, errors in the location of the atria inside the thorax under random rotations in the 3 axes were 7.9 \pm 10.7 mm (Fig. 4.B).

Furthermore, the ability of the proposed method to orientate the atria in its actual position when simultaneous rotations in 2 or 3 axes are present was evaluated. For that purpose, a random rotation in the $\mathrm{X}$ axis was applied $\left(-30^{\circ}\right.$ to $30^{\circ}$ ) and then the curvature was evaluated for rotations in the $\mathrm{Y}$ axis solving the location in the $\mathrm{Y}$ axis with an error of $8.9 \pm 7.6^{\circ}$ for mathematical models and $18.4 \pm 14.6^{\circ}$ for patients (Fig. 4.C). Finally, a random rotation was applied in the $\mathrm{X}$ and $\mathrm{Y}$ axes and then the curvature was evaluated for rotations in the $\mathrm{Z}$ axis. This case showed an error of $6.4 \pm 7.1^{\circ}$ in the angular deviation identification for mathematical models and $10.0 \pm 12.8^{\circ}$ in patients.

\section{Discussion}

This study presents a new approach for refining the location of the atria inside the thorax by solving the inverse problem of electrocardiography based on electrical 

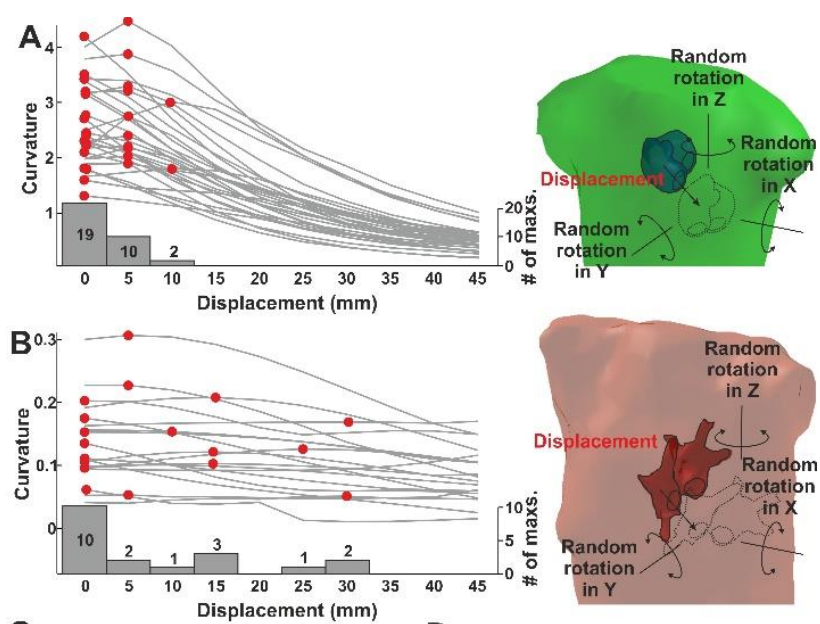

\section{C}
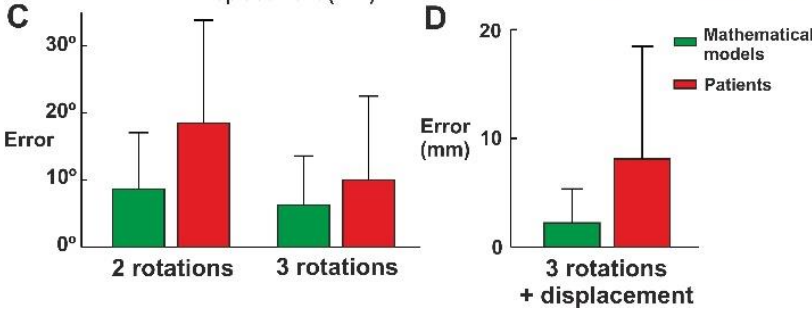

Figure 4. Estimation of accumulated artifacts: displacement with a random rotation in $\mathrm{X}, \mathrm{Y}$ and $\mathrm{Z}$ axes for (A) mathematical models and (B) patients. (C) Error in atrial orientation for cumulative rotations in 2 and 3 axes. (D) Error in atrial displacement with a random rotation in $\mathrm{X}, \mathrm{Y}$ and $\mathrm{Z}$ axis.

information, whose validity has been tested both in mathematical models and in patients. The L-curve regularization method has been profusely used in the literature to solve ill-posed systems, as the case of the inverse problem of electrocardiography [1]. Previous works have reported the sensitivity of the inverse problem to uncertainties on either the signals or the transfer matrix [7]. Our work makes use of the change in the L-curve curvature that occurs when there are inaccuracies in the transfer matrix, in our case caused by uncertainties in the atrial location, which cause significant errors in the inverse-computed EGMs. In this case, the division between the numerical error and the error on the solution (the two "arms" of the L-curve) is not so abrupt, thus it produces a smothered curvature in the "corner" of the Lcurve. However, this is the first work that proposes to take advantage of this property of the $\mathrm{L}$ curve in order to refine the location of the heart anatomy.

We have shown that the location of the atria can be resolved in a stepwise manner. An exhaustive search in the entire volume may be too computationally intense. However, the accuracy in the location of the atria may be increased if the algorithm was run more than once and thus the location was iteratively refined, since the errors in location or angular deviation are lower when no other inaccuracies are involved.

Finally, the validity of the method has been tested just for AF signals, its extension to other atrial arrhythmias remains unproven. However, AF presents the most complicated electrical pattern that can be found in cardiac electrophysiology, and sensible to the low-pass filtering effect of the torso conductor. We believe that the method could be extended to other less complicated.

This work presents a novel technique based on the curvature of the L-curve for placing the atria inside the chest when solving the inverse problem of electrocardiography. The presented technique has been tested for both mathematical models and patients, and under single or multiple uncertainties on the atrial location. It has been shown that this geometrical identification can be achieved with errors of about $1 \mathrm{~cm}$ and $15^{\circ}$. This technique could be useful for avoiding the spatial artifacts present in the inverse problem but also to extend the use of this technique by making possible the utilization of previously obtained CT/MRI images of the patient.

\section{Acknowledgements}

Generalitat Valenciana Grants (ACIF/2013/021); the Instituto de Salud Carlos III (PI13-01882, PI13-00903; PI14/00857; TEC2013-46067-R and DTS16/00160); Spanish Society of Cardiology (GCRC 2015) and Spanish Ministry of Science (Red RIC, PLE2009-0152).

\section{References}

[1] Horácek BM, Clements JC. The Inverse Problem of Electrocardiography: A Solution in Terms of Single- and Double-Layer Sources on the Epicardial Surface. Math Biosci 1997;144:119-54.

[2] Tikhonov AN. On the solution of incorrectly posed problems and the method of regularization. Sov Math Dokl 1963;4: 1035-1038.

[3] Dössel O, Krueger MW, et al. Computational modeling of the human atrial anatomy and electrophysiology. Med Biol Eng Comput 2012;50:773-99.

[4] Koivumäki JT, Seemann G, et al. In silico screening of the key cellular remodeling targets in chronic atrial fibrillation. PLoS Comput Biol 2012;10:e1003620.

[5] Pedrón-Torrecilla J, Rodrigo $M$, et al. Noninvasive Estimation of Epicardial Dominant High-Frequency Regions During Atrial Fibrillation. J Cardiovasc Electrophysiol 2016;27:435-42.

[6] Guillem MS, Climent AM, et al. Noninvasive localization of maximal frequency sites of atrial fibrillation by body surface potential mapping. Circ Arrhythm Electrophysiol 2013;6: 294-301.

[7] Ramanathan C, Rudy Y. Electrocardiographic imaging: II. Effect of torso inhomogeneities on noninvasive reconstruction of epicardial potentials, electrograms, and isochrones. J Cardiovasc Electrophysiol 2001;12:241-52.

Address for correspondence.

Miguel Rodrigo.

ITACA Institute, UPV.

Cami de Vera s/n 46022 Valencia. 